\title{
Motivation to quit smoking among hospitalised individuals with and without mental health disorders
}

\author{
Ranita Siru, Gary K. Hulse, Riaz J. K. Khan, Robert J. Tait
}

\begin{abstract}
Background: Persons with mental health disorders (MHD) have higher rates of smoking and poorer cessation of smoking outcomes than those without MHD. A decreased level of motivation may partially explain lower cessation rates, but there is little information on motivation among inpatients with MHD.

Objectives: Primary aims were to compare (1) motivation to cease smoking among those hospitalised with MHD or non-MHD, (2) the proportion that attempted smoking cessation, and (3) use of aids to cessation. A secondary aim was to assess cessation up to six months post-discharge.

Methods: Smokers were recruited at a tertiary hospital in Perth, Western Australia. Surveys were administered upon admission and at 5 and 14 days and 6 months post-discharge.

Results: We recruited $64 \mathrm{MHD}$ inpatients and 43 non-MHD inpatients. At baseline there were no significant differences between the groups on any measures of the five measures of motivation. Significantly more of the MHD sample attempted smoking cessation than those in the non-MHD sample (34 versus 13: $\chi^{2}(1)=5.472, P=0.028$ ). Nicotine replacement therapy (NRT) alone was used by $70 \%$ of those attempting to quit but was only provided as part of discharge medication to two people and few persons $(<21 \%)$ in either group used NRT post-discharge. By 14 days, three $(4.7 \%)$ of the MHD group and none $(0 \%)$ of the non-MHD group reported abstinence, at 6-months one from each group reported continuous abstinence since discharge from hospital.

Conclusions: Motivation to cease smoking among inpatients with MHD was similar to those without MHD, as was use of NRT while hospitalised. The low provision of post-discharge NRT may contribute to the poor cessation of smoking outcomes and does not fulfil evidence based guidelines.
\end{abstract}

Key words: smoking, inpatients, motivation, smoking cessation.

\section{Australian and New Zealand Journal of Psychiatry 2010; 44:640-647}

Dr Robert Tait, Research Fellow (correspondence)

School of Psychiatry \& Clinical Neurosciences, University of Western Australia, Queen Elizabeth II Medical Centre, Nedlands, Western Australia 6009, Australia and Centre for Mental Health Research, Australia National University, Canberra, ACT 0200, Australia. Email: Robert.tait@anu.edu.au

Ranita Siru, BMedSc Student; Gary K. Hulse, Winthrop Professor

School of Psychiatry \& Clinical Neurosciences, University of Western Australia, Queen Elizabeth II Medical Centre, Nedlands, WA 6009, Australia

Riaz J. K. Khan, Orthopaedic Surgeon

School of Surgery and Pathology, University of Western Australia, Queen Elizabeth II Medical Centre, Nedlands, WA 6009, Australia

Received 7 December 2009; accepted 13 January 2010.
Persons with mental health disorders (MHD) smoke at greater rates [1] and are more nicotine dependent [2] than the general population. This may contribute to the elevated rate of smoking-related illnesses in persons with MHD [3-5]. Cigarette smoking can also result in substantial decreases in the plasma level of some medications [6], with the associated loss of efficacy resulting in an increase in psychiatric symptoms, more frequent hospital admissions and a requirement for higher medication doses [7-9]. Persons with MHD may also endure significant social and financial deprivation in order to 
continue smoking [10] and individuals with MHD are only one-fifth as likely to cease smoking compared with the general population [11].

The elevated prevalence of smoking among persons with MHD may result from a lack of motivation to quit, or an inability to quit despite a desire to do so. Individuals with MHD appear to experience less success with quitting than individuals without MHD [12-14]. For instance, the presence of depressive symptoms during tobacco abstinence has been associated with failed quit attempts, an increased probability of returning to smoking, and the need for more quit attempts $[15,16]$. In trials of nicotine replacement therapy (NRT) and bupropion for smoking cessation in people with schizophrenia [1719], post-treatment abstinence rates of $11-13 \%$ were lower than the $17-35 \%$ rates reported by persons without schizophrenia [20]. Finally, among those with addictive disorders, smoking quit rates have been estimated to be four times lower than that of the general population [21]. People with MHD may have difficulty achieving and maintaining abstinence due to high levels of nicotine dependence [22,23], an increased severity of nicotine withdrawal symptoms compared to people without MHD [24], and a reliance on cigarettes as a form of self-medication [25].

Many mental health staff assume that persons with MHD are not motivated to quit smoking [26,27]. Despite this assumption, a recent review found that stage of change distributions were not significantly different for those with or without MHD, though it was noted that the majority of the reviewed studies were conducted in outpatients with MHD, rather than inpatients [28].

Further research among inpatients with MHD is warranted. Firstly, patients hospitalised with MHD are generally more unwell, and perhaps less able to make decisions about smoking cessation, than MHD outpatients. Secondly, as smoking bans become increasingly common in inpatient settings, mental health staff need to be aware of the proportion of smokers who are unmotivated to quit smoking, and potentially resistant to stopping smoking even briefly. Finally hospitalisation has been shown to be a teachable moment for smoking cessation among general medical patients $[29,30]$ and thus may be an opportune time to implement cessation interventions in motivated persons with MHD.

Therefore the primary aims of this study were to:

1. Describe and compare motivation to quit smoking in two groups of inpatients - those admitted for mental health disorders (MHD sample) and those admitted for orthopaedic or plastic surgery (non-MHD sample).
2. Identify the proportion of MHD or non-MHD patients who attempted smoking cessation during their inpatient stay, defined as commencement of either NRT or non-NRT cessation interventions.

3. Identify the proportions that elect to use different smoking cessation methods.

A secondary aim was to:

4. Compare the proportions of the MHD and nonMHD samples who successfully quit at 5 and 14 days and 6 months post-discharge.

\section{Methods}

\section{Setting}

Sir Charles Gairdner Hospital (SCGH) is a major teaching hospital in Western Australia. In 2007, SCGH implemented a non-smoking policy, which prohibited smoking by staff, patients and visitors anywhere on hospital grounds.

\section{Participants}

All participants were current smokers recruited from SCGH who could speak English and provide written consent. The MHD eligible population consisted of all smokers admitted to the Department of Psychiatry between March and August 2008. The non-MHD eligible population initially consisted of smokers admitted to the Department of Orthopaedic Surgery over the same period.

Orthopaedic Surgery patients were initially chosen as they were unlikely to be admitted with smoking-related illness, were likely to be hospitalised for periods similar to those observed in MHD patients, and were expected to have a similar distribution of ages to the MHD patients. Prior to the study, anonymous demographic and length of stay (LOS) data for 1 month of admissions (Orthopaedic Surgery $n=113$ : Psychiatry $n=159$ ) were compared. There was a significant Gender $\times$ Department effect on age and a main effect of Department on LOS, with orthopaedic patients having a shorter LOS. To reduce these disparities, females over 65 years and day-of-surgery admissions (LOS less than 24 hours) were excluded from the non-MHD sample when recruitment commenced.

Due to low recruitment rates from orthopaedics, additional participants were sought from the Department of Plastic Surgery from May 2008 onwards. These patients were chosen for the same reasons as orthopaedics patients were originally selected.

\section{Procedures}

The study comprised a series of face-to-face interviews using a standard set of questionnaires. Additionally, we extracted admission and discharge dates, medications, primary and secondary diagnoses and 
whether the participant was ever an involuntary patient during the admission, from medical records.

As soon as possible after admission, patients were approached by the researcher and their smoking status determined. Current smokers were invited to participate in the study. Written consent was obtained and the baseline questionnaire administered. Post-discharge surveys were conducted at five and fourteen days and six months after the patient's date of discharge.

This study was approved by the SCGH Research Ethics Committee.

\section{Measures}

\section{Baseline}

Demographics and smoking history. Information on age, gender and level of education were collected, as were age at smoking initiation, cigarette consumption per day, prior quitting experience and potential barriers to quitting. Level of nicotine dependence was assessed using the Fagerstrom Test of Nicotine Dependence (FTND) [31].

Motivation. Five different measures were used. The first was a modified Stage of Change algorithm adapted from the University of Western Australia Survey on Recreation and Health [32]. Using this measure, patients were divided into three groups: Pre-contemplation (not thinking about giving up smoking), Contemplation Future (thinking about giving up smoking but not in the next fortnight) and Contemplation Current (thinking about giving up smoking in the next fortnight).

As the stages of change model has received criticism in the past [33], motivation was also assessed using three single-item measures previously evaluated by Sciamanna et al. [34]. 'Want' was assessed with the question, 'How much do you want to quit smoking?' (not at all, somewhat, very much), 'Likely' was evaluated using the question 'How likely is it that you will stay off cigarettes after you leave the hospital' (not likely, somewhat likely, very likely) and 'Confidence' asked participants to 'Please rate your confidence on a scale from 0 to 100 where 0 means there's no chance of quitting and 100 means that you're confident about successfully quitting in the next month'. Finally, we asked participants about their intended smoking behaviour postdischarge. Possible responses were 'Return to smoking my usual amount or greater', 'Cut down on smoking my usual amount' and 'Continue not smoking'.

Cessation of smoking attempts. Participants were asked if they would use, had used or had received but not used NRT and/or nonnicotine replacement smoking cessation therapies during their inpatient stay. Prescription NRT was assessed by review of records. Smokers were considered to have made a cessation attempt if they received, used or were prescribed NRT or non-nicotine replacement therapies during their inpatient stay.

\section{Regular and post-discharge follow-up}

Regular follow-ups (every 3-5 days while in hospital) reassessed all measures of motivation, compliance and uptake of NRT. Post-discharge follow-ups assessed if and when participants had returned to smoking post-discharge, current cigarette consumption, all motivation measures except for 'Likely', and whether the participant had used any smoking cessation therapies since discharge.

\section{Analysis}

Descriptive statistics were used to report baseline demographics, smoking characteristics and motivation to quit smoking. Differences between groups were analysed using chi-square tests for categorical variables, or Fishers Exact Test where expected cell values fell below five. Independent samples t-tests were used to compare normally distributed continuous variables and non-parametric equivalents were used for other continuous variables.

\section{Results}

\section{Sample characteristics}

We recruited 107 smokers: 64 from Psychiatry and 43 from Orthopaedics/Plastic Surgery. Demographic and smoking history data are summarised in Table 1. The MHD and non-MHD samples were not significantly different in terms of age, gender or level of education. The non-MHD sample had a significantly shorter LOS and smoked less cigarettes per day while they were inpatients. Although the MHD sample had a higher median FTND score than the non-MHD sample ( 6.0 versus 4.0 ), usual cigarette consumption was not significantly different between the MHD and non-MHD samples. However, the MHD sample consumed significantly more cigarettes upon admission (18.6 versus 11.7$)$ and as inpatients (12.0 versus 0.0$)$ than the non-MHD sample.

Table 2 shows the primary and secondary (for 30 patients) psychiatric discharge diagnoses in the MHD group. The largest primary categories were personality disorders $(n=16)$ and substance use $(n=12)$. In all bar one instance, 'other' was used to code 'situational crises' or 'suicide ideation/attempt'. All participants were 'voluntary' admissions. Within the non-MHD sample, six participants had a MHD diagnosed during their admission: five with depression and one with dysthymia.

Follow-up interviews were conducted with $86(80 \%)$ people at 5 days, $81(76 \%)$ at 14 days and $46(43 \%)$ at 6 months (Table 1). Individuals lost to follow-up by 6 months were not significantly different in terms of age $(t(105)=1.32, P=0.187), \operatorname{LOS}(t(105)=1.73, P=0.087)$, usual number of cigarettes smoked at baseline $(U=1273.5, P=0.370)$ or group $\left(\chi^{2}(1)=0.460, P=0.498\right)$ from those who completed 6-month follow-ups.

\section{Motivation to quit smoking}

Of the MHD and non-MHD samples, 21 (32.8\%) and 18 (41.9\%), respectively, were not thinking about giving up smoking. When asked if they wanted to quit, however, only nine (14.1\%) of the MHD sample and eight (18.6\%) of the non-MHD sample responded 'not at all'. There were no significant differences between the groups on any of the measures of motivation (Table 3 ).

\section{Smoking cessation aids}

While in hospital $34(53.1 \%)$ of the MHD group attempted to quit compared with $13(30.2 \%)$ of the non-MHD group $\left(\chi^{2}(1)=5.472\right.$, $P=0.028)$ : of the current contemplators $13(65 \%)$ and eight $(50 \%)$ 


\begin{tabular}{|c|c|c|c|c|c|}
\hline \multicolumn{6}{|c|}{ Table 1. Sample characteristics and history of smoking } \\
\hline & & MHD (64) & Non-MHD (43) & Statistic & $\mathbf{P}$ \\
\hline Interviews - Baseline & $n(\%)$ & $64(100)$ & $43(100)$ & - & - \\
\hline $\begin{array}{l}\text { Interviews - 5-day } \\
\text { follow-up }\end{array}$ & $n(\%)$ & $48(75.0)$ & $38(88.4)$ & $\chi^{2}(1)=2.91$ & 0.088 \\
\hline $\begin{array}{l}\text { Interviews - 14-day } \\
\text { follow-up }\end{array}$ & $n(\%)$ & $46(71.9)$ & $35(81.4)$ & $\chi^{2}(1)=1.27$ & 0.260 \\
\hline $\begin{array}{l}\text { Interviews - 6-month } \\
\text { follow-up }\end{array}$ & $n(\%)$ & $27(42.2)$ & $21(48.8)$ & $\chi^{2}(1)=0.460$ & 0.498 \\
\hline Age (years) & Mean (SD) & $37.3(9.6)$ & $36.9(12.0)$ & $t(105)=0.193$ & 0.847 \\
\hline Gender (male) & $n(\%)$ & $34(53.1)$ & $29(67.4)$ & $\chi^{2}(1)=2.177$ & 0.140 \\
\hline Education (years) & median (IQR) & $11.5(10.0-13.9)$ & $11.0(10.0-12.0)$ & $U=1057.50$ & 0.061 \\
\hline LOS, days, & median (IQR) & $11.0(8.0-20.75)$ & $4.0(2.0-7.0)$ & $U=519.00$ & $<0.001 * *$ \\
\hline $\begin{array}{l}\text { Cigarettes per day - } \\
\text { Usual }\end{array}$ & median (IQR) & $14.3(10.0-22.8)$ & $12.5(5.7-22.5)$ & $U=1282.50$ & 0.551 \\
\hline $\begin{array}{l}\text { Cigarettes per day - } \\
\text { Admission }\end{array}$ & median (IQR) & $18.6(10.0-25.0)$ & $11.7(4.9-22.5)$ & $U=1041.50$ & $0.05 *$ \\
\hline $\begin{array}{l}\text { Cigarettes per day - } \\
\text { Inpatient }\end{array}$ & median (IQR) & $12.3(7.1-20.0)$ & $0(0.0-5.0)$ & $U=395.50$ & $<0.001 * *$ \\
\hline FTND & median (IQR) & $6.0(3.3-7.0)$ & $4.0(2.0-6.0)$ & $U=968.00$ & $0.009 * *$ \\
\hline Number quit attempts & median (IQR) & $3.0(2.0-5.5)$ & $2.0(2.0-3.0)$ & $U=1057.50$ & 0.179 \\
\hline $\begin{array}{l}\text { Longest quit attempt, } \\
\text { days }\end{array}$ & median (IQR) & $180.0(21.0-910.0)$ & $90.0(12.3-365.0)$ & $U=958.50$ & 0.229 \\
\hline
\end{tabular}

respectively attempted to quit smoking $\left(\chi^{2}(1)=0.82, P=0.364\right)$. Table 3 shows the main aids to cessation used by participants as inpatients. NRT patches and inhalers were the main forms of NRT used, with MHD participants more likely to receive/use these than the non-MHD participants (Table 3). 'Advice to cut down' was the main type of nonNRT received/used by participants in either group.

Only two individuals who received NRT as inpatients were prescribed NRT as a discharge medication, one from each group. At the 5-day follow-up, 10 (20.8\%) of the MHD sample and six (15.8\%) of the non-MHD sample had used NRT since discharge. Between the 5and 14-day follow-up, these had decreased to seven (15.2\%) and four $(11.8 \%)$ in the respective samples. At 6 months, five (18.5\%) from the

\section{Table 2. Discharge diagnoses in the MHD sample}

\section{Diagnosis}

Personality disorder

Substance use disorder

(including alcohol)

Depressive disorder

Psychotic disorder

Adjustment disorder

Anxiety disorder

Bipolar disorder

Other (e.g., suicide attempt, situational crisis)

\# All patients had a primary diagnosis, 30 had a secondary mental health diagnosis.
MHD group and four (19.0\%) from the non-MHD group reported using NRT, with three (11.1\%) and one (4.8\%), respectively, using non-NRT methods and five (18.5) and two (9.5\%) using both NRT and non-NRT aids.

\section{Abstinence and reduction in consumption post-discharge}

Assuming those lost to follow-up had returned to smoking, at the 5-day follow-up, five (10.4\%) of the MHD sample and three (7.9\%) of the non-MHD sample were abstinent (FET $p=0.999$ ). At the 14-day follow-up, these numbers had decreased to three $(4.8 \%)$ in the MHD sample and zero in the non-MHD sample (FET $P=0.273)$ and at 6 months one from each group reported not smoking since discharge (Table 4). (NB: the abstinent person from the non-MHD group was not interviewed at five or 14 days.)

Although the level of cigarette consumption by the non-MHD sample was significantly lower than cigarette consumption by the MHD sample at both 5- and 14-day follow-ups, Wilcoxon signedranks tests showed that significant declines in usual cigarette consumption occurred between baseline and 14-day follow-ups for both the MHD $(\mathrm{Z}=-2.43, P=0.015$, median -1.0 , IQR $-5.7,-0.0)$ and nonMHD samples $(Z=-4.00, P<0.001$, median -5.0 , IQR $-10.0,-0.8)$. However, by 6 months consumption was not significantly different from baseline levels.

\section{Discussion}

Previous comparisons of motivation to quit smoking between outpatients with MHD and the general population 
Table 3. Baseline measures of motivation and inpatient aids to cessation of smoking

\begin{tabular}{|c|c|c|c|c|c|}
\hline & & MHD & Non-MHD & Statistic & $P$ \\
\hline Stage of change, & $n(\%)$ & & & & \\
\hline $\mathrm{PC}$ & & $21(32.8)$ & $18(41.9)$ & & \\
\hline CF & & $23(35.9)$ & $9(20.9)$ & & \\
\hline $\mathrm{CC}$ & & $20(31.3)$ & $16(37.2)$ & $\chi^{2}(2)=2.79$ & 0.248 \\
\hline Want, & $n(\%)$ & & & & \\
\hline Not at all & & $9(14.1)$ & $8(18.6)$ & & \\
\hline Somewhat & & $24(37.5)$ & $20(46.5)$ & & \\
\hline Very much & & $31(48.4)$ & $15(34.9)$ & $\chi^{2}(2)=1.94$ & 0.379 \\
\hline Likely, & $n(\%)$ & & & & \\
\hline Not likely & & $19(29.7)$ & $15(34.9)$ & & \\
\hline Somewhat likely & & $27(42.2)$ & $21(48.8)$ & & \\
\hline Very likely & & $18(28.1)$ & 7 (16.3) & $\chi^{2}(2)=2.02$ & 0.365 \\
\hline Intent, & $n(\%)$ & & & & \\
\hline $\begin{array}{l}\text { Return to usual } \\
\text { amount or greater }\end{array}$ & & $20(31.3)$ & $14(32.6)$ & & \\
\hline Cut down & & $27(42.2)$ & $19(44.2)$ & & \\
\hline Continue not smoking & & $17(26.6)$ & $10(23.3)$ & $\chi^{2}(2)=0.15$ & 0.928 \\
\hline Confidence, median (IQR) & & $50(0.0-75.0)$ & $50(0.0-60.0)$ & $U=1296.50$ & 0.705 \\
\hline NRT - Patch & $n(\%)$ & $32(50.0)$ & $13(30.2)$ & $\chi^{2}(1)=4.12$ & 0.042 \\
\hline NRT - Inhaler & $n(\%)$ & $15(23.4)$ & $3(7.0)$ & $\chi^{2}(1)=4.98$ & 0.026 \\
\hline Any NRT $n(\%)$ & & $38(59.4)$ & $18(41.9)$ & $\chi^{2}(1)=3.16$ & 0.075 \\
\hline Advice to cut down & $n(\%)$ & $13(20.3)$ & $4(9.3)$ & $\chi^{2}(1)=2.33$ & 0.127 \\
\hline Any non-NRT & $n(\%)$ & $22(34.4)$ & $8(18.6)$ & $\chi^{2}(1)=3.17$ & 0.075 \\
\hline
\end{tabular}

have consistently found no difference in motivation between groups [26]. Until recently, however, studies assessing motivation to quit smoking among hospitalised patients have generally excluded individuals admitted for MHD [35-39]. The present study is therefore one of the first to assess and directly compare motivation to quit in hospitalised smokers with and without MHD. Notably, while in hospital, significantly more of the MHD group attempted the cessation of smoking, although there was no difference in the proportions abstinent post-discharge.

Current preconceptions suggest that few individuals with MHD are motivated to quit smoking, particularly individuals hospitalised with MHD. In this study, however, two-thirds of individuals in the MHD sample were contemplating quitting, some within the next 2 weeks. Furthermore, $50 \%$ of individuals with MHD stated that they wanted to quit smoking 'very much' and nearly $30 \%$ intended to continue not smoking post-discharge. Over $50 \%$ attempted cessation and of those who reported contemplating quitting in the next two weeks, $65 \%$ attempted cessation while hospitalised, a greater proportion than reported in the literature [40-42] and greater than the $50 \%$ in the non-MHD sample.

Despite the higher level of nicotine dependence among those with MHD and the lack of post-discharge cessation support, there was no significant difference between the
MHD and non-MHD samples in the numbers of individuals abstinent at each follow-up. These results are at odds with the assumption that persons with MHD are unwilling to attempt to quit smoking. This notwithstanding, in general, smokers with MHD do appear to have more difficulty maintaining long-term abstinence than smokers without MHD; at 6- and 12-month follow-ups, smokers with schizophrenia [22] and alcohol use disorder [43], respectively, reported significantly lower abstinence rates than individuals without MHD.

Given that more than half of the MHD group were motivated to attempt cessation, even in the absence of a formal cessation program, motivated individuals with MHD may use hospitalisation to initiate a cessation attempt. Indeed, by improving access to smoking cessation resources, and by enabling the organisation and coordination of long-term follow-up, hospitalisation may provide an ideal opportunity to begin smoking cessation in this otherwise neglected population of smokers $[29,36,38]$. Unfortunately, relapse immediately after discharge was high; nearly $90 \%$ of the MHD sample and $80 \%$ of the non-MHD sample had relapsed to smoking within 5 days of discharge. In another study, $76 \%$ of patients discharged from a psychiatric unit resumed smoking on the day of discharge, with the median time to first cigarette being 5 minutes [44]. 


\begin{tabular}{|c|c|c|c|c|c|}
\hline \multicolumn{6}{|c|}{ Table 4. Cigarette consumption at 5- and 14-day and 6-month follow-ups } \\
\hline & & MHD & Non-MHD & Statistic & $P$ \\
\hline Abstinent for 5 days & $n(\%)$ & $5(7.8)$ & $3(7.0)$ & FET & 0.999 \\
\hline Abstinent for 14 days & $n(\%)$ & $3(4.7)$ & $0(0.0)$ & FET & 0.273 \\
\hline Abstinent for 6 months & $n(\%)$ & $1(1.6)$ & $1(2.3)$ & FET & 0.999 \\
\hline $\begin{array}{l}\text { Abstinent at } 6 \text { months } \\
\quad \text { (last } 4 \text { weeks) }\end{array}$ & $n(\%)$ & $4(6.3)$ & $1(2.3)$ & FET & 0.646 \\
\hline CPD at 5-day follow-up, & median (IQR) & $11.4(5.0-24.4)$ & $8.3(1.5-13.1)$ & $U=649.0$ & 0.022 \\
\hline CPD at 14-day follow-up, & median (IQR) & $12.0(9.9-20.0)$ & $8.0(2.9-15.0)$ & $U=480.5$ & $0.006^{* * *}$ \\
\hline CPD at 6-month follow-up, & median (IQR) & $15.0(10.0-20.0)$ & $15.0(10.0-20.0)$ & $U=212.0$ & 0.503 \\
\hline
\end{tabular}

The lack of hospital staff follow-up for patients attempting to quit, and the poor rate of usage of NRT at follow-ups may have contributed to poor outcomes in both the MHD and non-MHD sample. Clinical practice guidelines recommend that a minimum three-day supply of NRT be provided at discharge to smokers intending to quit or remain abstinent [45]. A recent Cochrane review proposed that hospital-based interventions incorporate a behavioural component and include at least 1 month of supportive contact post-discharge [46]. In this study, although 35 participants intended to continue not smoking post-discharge, only two received NRT as a discharge medication.

Provision of NRT both during and after a period of hospitalisation may be particularly important for motivated smokers with MHD, as socioeconomic deprivation is common in this population [47] and may prevent these individuals from accessing smoking cessation aids [48]. Smokers with MHD may also require extended-duration or high-dose NRT as a result of their high-level of nicotine dependence [22]. Within the mental health setting, many of the structures necessary for the successful implementation of hospital-based smoking cessation interventions are already in place. Importantly, and unlike most patients hospitalised for other ailments, many mental health inpatients, particularly those with psychotic disorders, are followed-up for lengthy periods as outpatients. Others are referred to general practitioners or to specialist alcohol or other drug services, allowing smoking cessation to be integrated into continuing psychiatric care.

Given that inpatients with MHD appear to be both as motivated and as capable of initiating quitting smoking as inpatients without MHD, other explanations for the persistently elevated prevalence of smoking in this cohort must be sought. The lack of encouragement and support by mental health staff may be one reason that persons with MHD fail at smoking cessation. In the present study, only $20 \%$ of smokers with MHD reported receiving advice to cut down. Other studies have found that although psychiatrists and psychiatric trainees ask about their patients' smoking status and give smokers advice to quit, assistance to quit is offered by less than one-fifth of mental health professionals, possibly because they feel inadequately trained to deal with smoking cessation or lack confidence in their ability to help patients quit. Mental health staff may also suffer from 'therapeutic nihilism' and feel discouraged from promoting cessation by the perception that their clients are uninterested in quitting and unresponsive to suggestions to quit $[27,49]$. In addition, the high prevalence of smoking among mental health professionals, such as psychiatric nurses, compared to nurses in other areas, may also impede cessation of smoking programs [50]. Therefore, in addition to clear guidelines and structured smoking cessation programs, it is imperative that mental health staff receive education and training to make them aware that their clients are receptive and responsive to such interventions.

\section{Limitations}

Data collection from a single study site limits the generalisability of the findings to other settings. The sample size was modest, so the power to detect differences between groups may have been limited. The questionnaire addressed potentially sensitive topics such as alcohol and tobacco use and non-compliance with hospital regulations, thus the possibility of social desirability bias cannot be excluded. However, the participants displayed little reluctance in admitting to non-compliance with the non-smoking policy, thus it is hoped that this openness extended to other aspects of the questionnaire.

Six of the non-MHD sample had a MHD diagnosed during admission, and the prevalence of past or remitted MHD in the non-MHD sample was not known. The failure to exclude participants with active MHD from 
the non-MHD sample may have introduced a confounding factor. The low rate of recruitment in the non-MHD group was of surprise and this may reflect the low prevalence of smoking among eligible patients of the Departments of Orthopaedic and Plastic Surgery. Additionally, that this was a research project rather than a clinical intervention may have also reduced participation, as participants were not obviously receiving any benefit, such as free NRT, from the study.

Finally, differences between the stage of change algorithms used in this and other studies [51], restrict the comparability of the findings of this study. Compared to the Preparation stage of change outlined by DiClemente et al. [51], however, the Contemplation Current stage of change may be an even stricter measure of readiness to quit, since persons in the Contemplation Current stage must be considering quitting within the next 2 weeks, rather than the next month. Thus, this measure was likely to underestimate, rather than overestimate, motivation, which only strengthens the finding that persons with MHD are highly motivated to quit.

\section{Conclusion}

This study demonstrates that many individuals with MHD are motivated to quit smoking, seek support with cessation and are receptive to cessation interventions, even while hospitalised. Moreover, after a period of hospitalisation, the proportion of patients able to achieve short-term abstinence was not significantly different for those with or without MHD. In contrast, many mental health staff assume that their clients are unmotivated and unable to quit smoking. Despite the existence of clinical practice guidelines which advocate the provision of smoking cessation services to persons with MHD [52], these guidelines are not being universally implemented. Education and training for staff will be necessary before hospital-based smoking cessation interventions, incorporating NRT, behavioural counselling and long-term outpatient follow-up, can be properly implemented.

\section{Acknowledgements}

We thank the participants and staff at SCGH for their help in this study.

Declaration of interest: None to declare. This research was funded by an A\&A Saw Scholarship awarded to RS by the University of Western Australia.

\section{References}

1. Lasser K, Boyd JW, Woolhandler S, Himmelstein DU, McCormick D, Bor DH. Smoking and mental illness: A population-based prevalence study. JAMA 2000; 284:2606-2610.

2. Dani JA, Harris RA. Nicotine addiction and comorbidity with alcohol abuse and mental illness. Nature Neurosci 2005; 8:1465-1470.

3. Baker A, Richmond R, Haile M, et al. A randomized controlled trial of a smoking cessation intervention among people with a psychotic disorder. Am J Psychiatry 2006; 163:1934-1942.

4. Dalton SO, Mellemkjaer L, Olsen JH, Mortensen PB, Johansen C. Depression and cancer risk: A register-based study of patients hospitalized with affective disorders, Denmark, 1969-1993. Am J Epidemiology 2002; 155:1088-1095.

5. Lawrence DM, Holman CDAJ, Jablensky AV, Hobbs MST. Death rate from ischaemic heart disease in Western Australian psychiatric patients 1980-1998. Br J Psychiatry 2003; 182:31-36.

6. Williams JM, Ziedonis D. Addressing tobacco among individuals with a mental illness or an addiction. Addictive Behav 2004; 29:1067-1083.

7. Dalack GW, Glassman AH. A clinical approach to help psychiatric patients with smoking cessation. Psychiatr $Q$ 1992; 63:27-39.

8. Desai HD, Seabolt J, Jann MW. Smoking in patients receiving psychotropic medications: A pharmacokinetic perspective. CNS Drugs 2001; 15:469-494.

9. Goff D, Henderson D, Amico B. Cigarette smoking in schizophrenia: Relationship to psychopathology. Am J Psychiatry 1992; 149:1189.

10. Lawn SJ, Pols RG, Barber JG. Smoking and quitting: A qualitative study with community-living psychiatric clients. Soc Sci Med 2002; 54:93-104.

11. Diaz FJ, Rendon DM, Velasquez DM, Susce MT, de Leon J. Datapoints: Smoking and smoking cessation among persons with severe mental illnesses. Psychiatr Serv 2006; 57:462.

12. Breslau N, Klein DF. Smoking and panic attacks: An epidemiologic investigation. Arch Gen Psychiatry 1999; 56:1141-1147.

13. Centers for Disease Control and Prevention (CDC). Efforts to quit smoking among persons with a history of alcohol problems: Iowa, Kansas, and Nebraska, 1995-1996. MMWR Morb Mort Wkly Rep 1997; 46:1144-1148.

14. Niaura R, Britt DM, Borrelli B, Shadel WG, Abrams DB, Goldstein MG. History and symptoms of depression among smokers during a self-initiated quit attempt. Nicotine Tob Res 1999; 1:251.

15. Glassman AH. Cigarette smoking: Implications for psychiatric illness. Am J Psychiatry 1993; 150:546-553.

16. Wagena EJ, Kant I, van Amelsvoort LGPM, Wouters EFM, van Schayck CP, Swaen GMH. Risk of depression and anxiety in employees with chronic bronchitis: The modifying effect of cigarette smoking. Psychosom Med 2004; 66:729-734.

17. Addington J, el-Guebaly N, Campbell W, Hodgins DC, Addington D. Smoking cessation treatment for patients with schizophrenia. Am J Psychiatry 1998; 155:974-975.

18. Evins AE, Mays VK, Rigotti NA, Tisdale T, Cather C, Goff DC. A pilot trial of bupropion added to cognitive behavioral therapy for smoking cessation in schizophrenia.Nicotine Tob Res 2001; 3:397-403.

19. Weiner E, Ball MP, Summerfelt A, Gold J, Buchanan RW. Effects of sustained-release bupropion and supportive group therapy on cigarette consumption in patients with schizophrenia. Am J Psychiatry 2001; 158:635-637.

20. Hughes JR, Goldstein MG, Hurt RD, Shiffman S. Recent advances in the pharmacotherapy of smoking. JAMA 1999; 281: $72-76$. 
21. Richter KP, Gibson CA, Ahluwalia JS, Schmelzle KH. Tobacco use and quit attempts among methadone maintenance clients. Am J Pub Health 2001; 91:296-299.

22. Horst WD, Klein MW, Williams D, Werder SF. Extended use of nicotine replacement therapy to maintain smoking cessation in persons with schizophrenia. Neuropsychiatr Dis Treat 2005; 1:349-355.

23. Kinnunen T, Doherty K, Militello FS, Garvey AJ. Depression and smoking cessation: Characteristics of depressed smokers and effects of nicotine replacement. J Consult Clinical Psychol 1996; 64:791-798.

24. Xian H, Scherrer JF, Madden PA, et al. Latent class typology of nicotine withdrawal: Genetic contributions and association with failed smoking cessation and psychiatric disorders. Psychol Med 2005; 35:409-419.

25. Pomerleau CS, Marks JL, Pomerleau OF. Who gets what symptom? Effects of psychiatric cofactors and nicotine dependence on patterns of smoking withdrawal symptomatology. Nicotine Tob Res 2000; 2:275-280.

26. Etter M, Mohr S, Garin C, Etter J-F. Stages of change in smokers with schizophrenia or schizoaffective disorder and in the general population. Schizophr Bull 2004; 30:459-468.

27. Price J, Ambrosetti L, Sidani J, Price J. Psychiatrists' smoking cessation activities with Ohio community mental health center patients. Community Ment Health $J$ 2007; 43:251-266.

28. Siru R, Hulse GK, Tait RJ. Assessing motivation to quit smoking in persons with mental illness: A review. Addiction 2009; 104: 719-733.

29. Orleans CT, Kristeller JL, Gritz ER. Helping hospitalized smokers quit: New directions for treatment and research. J Consult Clinical Psychol 1993; 61:778-789.

30. Rigotti NA, McKool KM, Shiffman S. Predictors of smoking cessation after coronary artery bypass graft surgery: Results of a randomized trial with 5-year follow-up. Ann Intern Med 1994; 120:287-293.

31. Heatherton TF, Kozlowski LT, Frecker RC, Fagerstrom K-O. The Fagerstrom Test for Nicotine Dependence: a revision of the Fagerstrom Tolerance Questionnaire. Br J Addiction 1991; 86: $1119-1127$.

32. Clarkson J, Donovan R, Giles-Corti B, Bulsara M, Jalleh G. Survey on recreation and health 1992-1998: Executive Overview, In: Health Promotion Evaluation. Unit Department of Public Health, The University of Western Australia. Perth, 2000.

33. West R. Time for a change: Putting the Transtheoretical (Stages of Change) Model to rest. Addiction 2005; 100:1036-1039.

34. Sciamanna CN, Hoch JS, Duke C, Fogle MN, Ford DE. Comparison of five measures of motivation to quit smoking among a sample of hospitalized smokers. J Gen Int Med 2000; $15: 16-23$

35. Lando H, Hennrikus D, McCarty M, Vessey J. Predictors of quitting in hospitalized smokers. Nicotine Tob Res 2003; 5:215-222.

36. Lewis SF, Piasecki TM, Fiore MC, Anderson JE, Baker TB. Transdermal nicotine replacement for hospitalized patients: A randomized clinical trial. Prev Med 1998; 27:296-303.
37. MacKenzie TD, Pereira RI, Mehler PS. Smoking abstinence after hospitalization: Predictors of success. Prev Med 2004; 39: 1087-1092.

38. Ong KC, Cheong GN, Prabhakaran L, Earnest A. Predictors of success in smoking cessation among hospitalized patients. Respirology $2005 ; 10: 63-69$.

39. Rigotti NA, Arnsten JH, McKool KM, Wood-Reid KM, Singer DE, Pasternak RC. The use of nicotine-replacement therapy by hospitalized smokers. Am J Prev Med 1999; 17:255-259.

40. Carosella AM, Ossip-Klein DJ, Owens CA. Smoking attitudes, beliefs, and readiness to change among acute and long term care inpatients with psychiatric diagnoses. Addictive Behav 1999; 24:331-344.

41. Hall RG, Duhamel ML, McClanahan RL, et al. Level of functioning, severity of illness, and smoking status among chronic psychiatric patients. J Nerv Ment Dis 1995; 183:468-471.

42. Lucksted A, McGuire C, Postrado L, Kreyenbuhl J, Dixon LB. Specifying cigarette smoking and quitting among people with serious mental illness. Am J Addict 2004; 13:128-138.

43. Hughes JR. Treatment of smoking cessation in smokers with past alcohol/drug problems. J Subst Abuse Treat 1993; 10:181-187.

44. Prochaska JJ, Fletcher L, Hall SE, Hall SM. Return to smoking following a smoke-free psychiatric hospitalisation .Am J Addict 2006; 15:15-22.

45. New South Wales Department of Health. Guide for the management of nicotine dependent inpatients - Flowchart, Sydney 1 January 2002.

46. Rigotti NA, Munafo MR, Stead LF. Interventions for smoking cessation in hospitalised patients. Cochrane Database Syst Rev 2007; DOI; 1002/14651858.CD001837.pub2.

47. Davidson S, Judd F, Jolley D, Hocking B, Thompson S. The general health status of people with mental illness. Australas Psychiatry 2000; 8:31-35.

48. Siahpush M, McNeill A, Borland R, Fong GT. Socioeconomic variations in nicotine dependence, self-efficacy, and intention to quit across four countries: Findings from the International Tobacco Control (ITC) Four Country Survey. Tob Control 2006; 15:iii $71-75$.

49. Prochaska JJ, Fromont SC, Hall SM. How prepared are psychiatry residents for treating nicotine dependence? Acad Psychiatry 2005; 29:256-261.

50. Sarna L, Bialous SA, Wells MJ, Kotlerman J, Froelicher ES, Wewers ME. Do you need to smoke to get a break?: Smoking status and missed work breaks among staff nurses. Am J Prev Med 2009; 37:S165-171.

51. DiClemente CC, Prochaska JO, Fairhurst SK, Velicer WF, Velasquez MM, Rossi JS. The process of smoking cessation: An analysis of precontemplation, contemplation, and preparation stages of change. J Consult Clinical Psychol 1991; 59: 295-304.

52. American Psychiatric Association. Practice guideline for the treatment of patients with nicotine dependence. Am J Psychiatry 1996; 153:1. 